\title{
3D laparoscopy as a tertiary cytoreductive (TCR) surgery in infiltrating ureter recurrent epithelial ovarian cancer - case report and a mini-review of the literature
}

\author{
Tomasz Szopiński ${ }^{1}$, Igal Mor ${ }^{1}$, Krzysztof Okoń², Włodzimierz Baranowski ${ }^{3}$, Małgorzata Jerzak ${ }^{1}$ \\ ${ }^{1}$ Mazovia Hospital, Warsaw, Poland \\ ${ }^{2}$ Department of Pathomorphology, Jagiellonian University Medical College, Krakow, Poland \\ ${ }^{3} 1^{\text {st }}$ Department of Obstetrics and Gynaecology, $1^{\text {st }}$ Faculty of Medicine, Medical University of Warsaw, Warsaw, Poland
}

\begin{abstract}
The paper describes a case of a 61-year-old woman with recurrent epithelial ovarian cancer infiltrating the ureter treated with 3D laparoscopy as a tertiary cytoreductive surgery (TCR). In addition, a mini-review of the literature concerning TCR is presented.
\end{abstract}

Key words: cytoreduction, recurrent ovarian cancer, 3D laparoscopy.

\section{Introduction}

Currently, ovarian cancer is the sixth most common cancer worldwide among women in developed countries and the most lethal of all gynaecologic malignancies [1]. Unfortunately, most women have advanced-stage disease at the time of diagnosis [2]. Complete cytoreductive surgery is necessary for significant survival benefit [3]. Recent data suggest that even tertiary cytoreductive (TCR) surgery in recurrent epithelial ovarian cancer (EOC) to achieve no residual disease postoperatively is associated with significantly longer overall survival in selected patients [4]. However, data regarding metastases to the urinary tract system are rather scarce. Metastatic involvement of the urinary tract in patients with advanced ovarian carcinoma were evaluated during autopsy. The distribution of metastatic sites was as follows: kidney 3.5\%, urinary bladder $22.4 \%$ and ureter $11.8 \%$ [5].

\section{Case report}

We present a case of a 61-year-old woman with recurrent ovarian cancer infiltrating the right ureter. It is 10 years since her primary operation with a diagnosis of FIGO (International Federation of Gynecology and Obstetrics) stage IIB EOC (epithelial ovarian cancercystadenocarcinoma solidum, partim papillare serosum ovariorum). After the primary operation 6 courses of adjuvant chemotherapy based on paclitaxel and the platinum analogue carboplatin were introduced.

Routine preoperative examination was performed in the study patient. Computed tomography (CT) scans of the chest, abdomen, and pelvis and tumour markers (CEA, CA19-9, and CA125) were obtained. Definitive primary cytoreductive surgery was carried out to achieve complete cytoreduction using the following procedures: laparotomy, abdominal wall resection, abdominal and pelvic lymphadenectomy, appendectomy, and bilateral adnexectomy with hysterectomy, cytoreductive surgery and biopsy of peritoneal implants, enterolysis, ureterolysis, and omentectomy. The peritonectomy procedures also include diaphragmatic, parietal, and pelvic peritonectomy, but it was not necessary in this case. Resection of hollow viscus and/or organs is also performed if they cannot be cleared of disease or were affected by the primary cancer, but these procedures were not necessary in this case and every effort was made to avoid extensive small bowel resection and/or stoma formation to preserve quality of life. Complete cytoreduction was defined as nodules less than $2.5 \mathrm{~mm}$ in size $(C C=1)$ or the absence of visible tumour nodules $(C C=0)$ and complete cytoreduction was achieved during primary surgery. However, after 8 years recurrence of the ovarian cancer was diagnosed and during the secondary surgery two tumours infiltrating the right iliac vessels 
and pelvis wall (diameter $3 \mathrm{~cm}$ ), with pressure to the right ureter, and peritoneum of the caecum (diameter $2 \mathrm{~cm}$ ). According to intraoperative inspection resection of the greater tumour was incomplete but the caecum metastasis was completely removed. Histological examination of the specimens was as follows: adenocarcinoma papillare serosum ex ovario CK7 (+), CK20 (-), WT-1 (+). According to urologist consultation a double-J (2JJ) catheter was placed after surgery to protect the function of the right kidney. However, the CT scan after surgery was normal based on RECIST criteria and the patient received 6 courses of adjuvant chemotherapy based on paclitaxel and carboplatin. Unfortunately, 2 years later the patient was admitted to perform surgery in order to remove the ovarian tumour recurrence located in the small pelvis. This tumour infiltrated the dilated right ureter above the bladder, causing its complete obstruction and right-sided hydronephrosis (Figs. 1 and 2). For this reason, a 2JJ catheter (pigtail) was placed before surgery to protect the function of the right kidney. In the CT examination with contrast, the cortical layer of this kidney was visible in the parenchymal phase without impaired urine transport to the bladder. The operation was performed laparoscopically using the Olympus Viscera Visual Track with 3D visualization. After the catheterization of the urinary bladder insufflation was created near the navel access to the peritoneal cavity by minilaparotomy through the $10 \mathrm{~mm}$ optical port. The patient was then placed in a Trendelenburg position with an angle of 22 degrees. The 4 ports $(3-5 \mathrm{~mm}$ and $1-10 \mathrm{~mm})$ were placed in a typical way for pelvic urological operations (Fig. 3). The ureter was initially identified at the height of the iliac vessels after peritoneal incision. Afterwards, the retroperitoneal space on the right side was exposed, cutting the peritoneum along the iliac vessels and then over the pubic symphysis. For better identification of the uri- nary bladder, it was temporarily filled with $0.9 \%$ sterile saline solution. Following the ureter, a change outside the bladder location was clearly identified. This tumour did not infiltrate the bladder wall and was easily bleeding when touched. Cutting $2 \mathrm{~cm}$ above the ureteral lesion and the tumour removal sharply in the range of macroscopically unchanged tissues were performed. The tumour was removed by stump and blind dissection with $2 \mathrm{~cm}$ of infiltrated ureter. Then the specimen was put into an endobag and removed. Then, the ureteral stump was transplanted into the bladder using a psoas high manoeuvre without producing an outflow mechanism. The anterior and lateral wall of the bladder was released so that it reached the ilium-lumbar muscle on the right side. With two sutures, the bladder was pulled up and attached. Then, in the top a longitudinal bladder incision and adequate ureteral spatulation were performed. Single monofilament sutures were used endto-end to perform anastomosis. A new 2JJ catheter was introduced into the ureter. The bladder was closed by running suture arch and water tightness was checked at $100 \mathrm{ml}$. Haemostasis control was performed. A drain was left in the area of the anastomosis. The bladder catheter was left for 3 weeks. On the $4^{\text {th }}$ day after the surgery, the drain, which had received a trace amount of serous content, was removed. After 6 weeks, the 2JJ catheter was removed. The post-operative CT scan revealed proper functioning of the right kidney and the free passage of contrasted urine into the bladder.

After the operation histopathology of the tumour was performed. The specimen consisted of fibromuscular tissue with infiltrate of moderately to poorly differentiated adenocarcinoma. The tumour formed papillary and tubular structures lined by cylindrical cells with a high nuclear-cytoplasmic ratio and nuclear atypia. Focally the epithelium was more multi-layered with slight similarity to urothelium. There were small areas of ne-

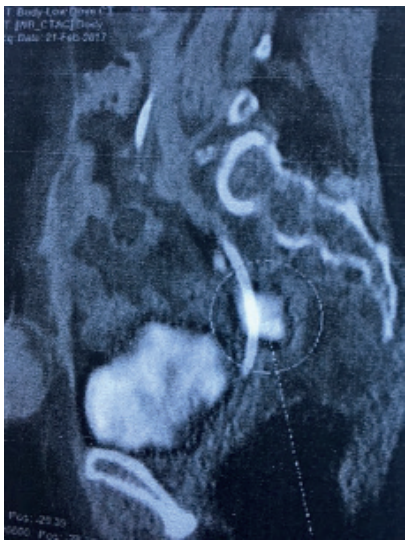

Fig. 1. Tumour infiltrating right ureter, PET-CT scan of the pelvis

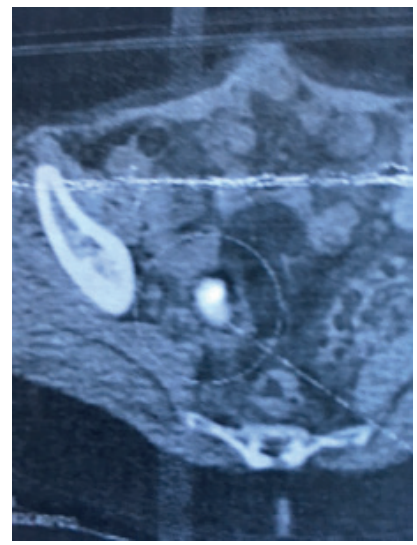

Fig. 2. Tumour infiltrating right ureter, PET-CT scan of the pelvis

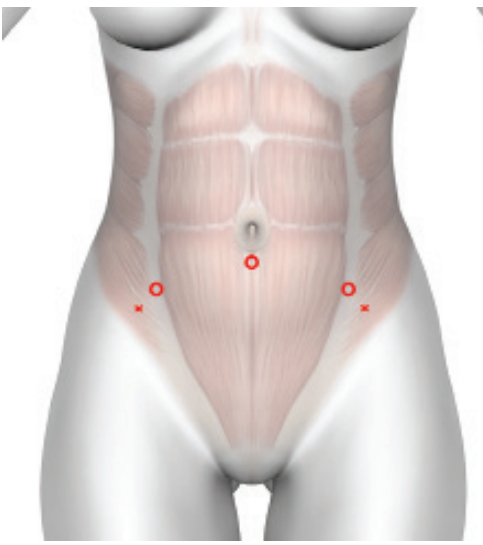

Fig. 3. The ports' location for pelvic urological operations 
crosis and significant mitotic activity. On immunohistochemistry, the tumour cells expressed WT1 and oestrogen receptor, but were negative for GATA3. Basing on these finding and on the case history, the diagnosis of relapsing high grade serous ovarian carcinoma could be established (Figs. 4-6).

Finally, according to the BRCA-1 + status in the specimen after the final operation the patient was assigned to receive maintenance therapy with olaparib. In addition, BRCA-1 mutation was negative in the blood of the study patient. CT scans did not reveal any abnormalities. At the moment, it is more than one year after the patient's TCR surgery and she feels fine.

\section{Discussion}

Recently, complete en bloc tumour resection with the right external and internal iliac artery and vein, right ureter, vagina, and rectum adhering to the tumour has been described. The authors concluded that exfoliation from the sacral plexus and total resection with external and internal iliac vessels enables complete resection of the tumour fixed to the pelvic sidewall [6]. Surgical procedures involving the urinary tract may be necessary to achieve optimal surgery in patients with ovarian peritoneal carcinomatosis with reasonable rates of postoperative morbidity $[4,6,7]$. Proper patient selection is the most important to have a chance of complete cytoreduction $[4,6,7]$. In the study case, the quality of life has been taken into account during the entire therapy. Modern management of recurrent ovarian cancer compromises various combinations of systemic chemotherapy with or without targeted agents since most patients with advanced-stage epithelial ovarian cancer will experience a relapse of disease despite a complete response after surgery and platinum-based chemotherapy [3]. Therefore, recent data suggest that olaparib (PARP inhibitor) provides a significant progression-free survival improvement with no detrimental effect on quality of life in patients with platinum-sensitive relapsed ovarian cancer and a BRCA1/2 mutation [8]. Another drug, prexasertib, a cell cycle checkpoint kinase 1 and 2 inhibitor, also showed clinical activity in BRCA wild-type high-grade serous ovarian carcinoma [9]. Olaparib was used as a maintenance therapy in the study patients according to the national program in Poland. WT1 is a useful immunohistochemical marker in diagnosis of high-grade serous carcinoma. The sensitivity and specificity for diagnosis of high-grade serous carcinoma were found to be 96 and 100\%, respectively [10]. Therefore, optimal surgery, chemotherapy and histopathology are the key steps leading to success in the therapy of relapsed ovarian cancer.

Despite the effect of various tumour biological factors such as grading and histological subtype, the surgical outcome is still the most important prognostic

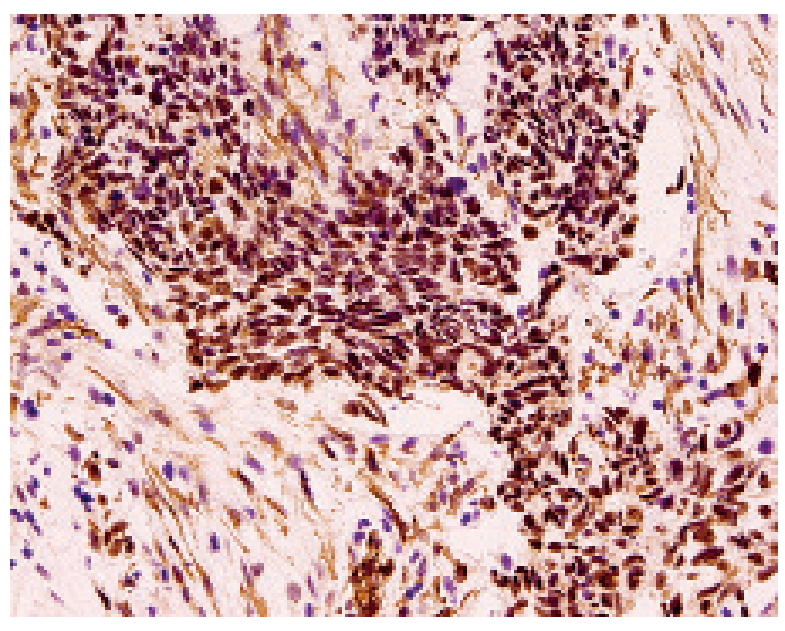

Fig. 4. Papillary and tubular architecture of the tumour. Haematoxylin and eosin, 100x

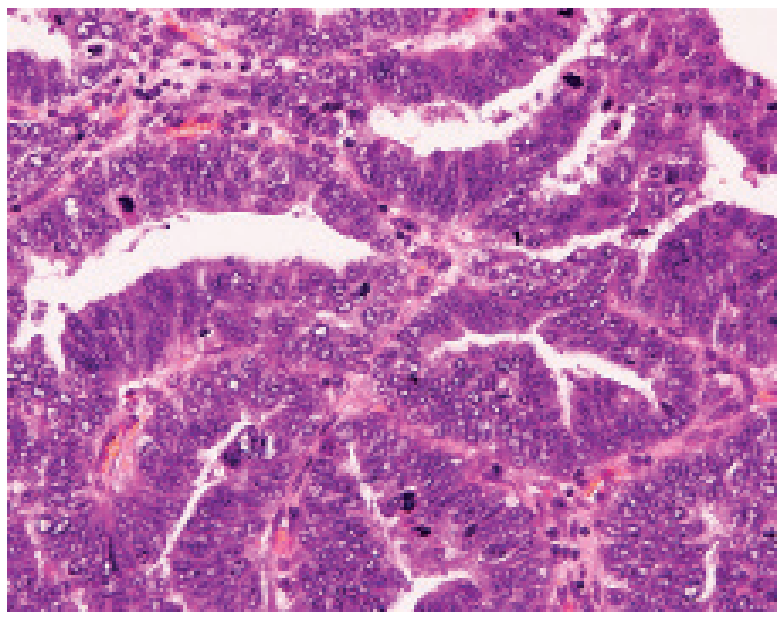

Fig. 5. Prominent cellular atypia with high nuclear-cytoplasmic ratio, pleomorphic and mitotic figures. Haematoxylin and eosin, $400 \times$

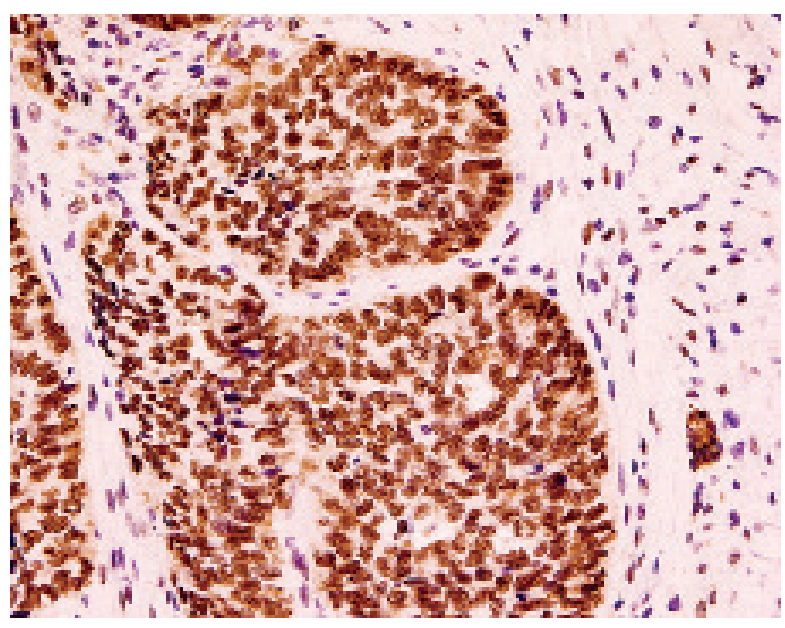

Fig. 6. Strong nuclear reaction for WT1. Immunohistochemistry, $200 \times$

factor for both progression-free and overall survival [11]. However, the management of cancer has remained a subject of an international discussion. Therefore, only 
a few prospective studies have focused on the effect of surgery in relapsed ovarian cancer. The impact of TCR on survival in EOC and predictors of complete cytoreduction were analysed in patients with $\mathrm{a} \geq 6$-month treatment-free interval (TFI), recently. Almost $70 \%$ of the patients achieved complete cytoreduction with severe post-operative complications in less than $10 \%$ and no cases of mortality within 60 days from surgery [4]. It has been established that women who underwent primary complete cytoreduction are in good performance status, and those who have only a minimal ascites volume (less than $500 \mathrm{ml}$ ) in the recurrent situation have $76 \%$ likelihood of undergoing complete resection and survival prolongation. The benefit seems to be greater in patients with TFI > 12 months showing a single-site recurrence disease, in which complete cytoreduction is achievable [12]. Therefore, preoperative assessment of patients and weighing the potential survival benefit against potential surgical risks are very important for patient selection [13]. In addition, postoperative tumour residual disease remains the strongest predictor of survival in TCR [14].

\section{Conclusions}

In conclusion, the challenge for the future is multidisciplinary cooperation between an oncological gynaecologist, urologist and clinical oncologist in recurrent ovarian cancer management involving metastases to the urinary system.

\section{Disclosure}

The authors report no conflict of interest.

\section{References}

1. Jemal A, Bray F, Center MM, et al. Global cancer statistics. CA Cancer J Clin 2011; 61: 69 Y90.

2. Modugano F, Edwards RP. Ovarian cancer: prevention, detection, and treatment of the disease and its recurrence. Molecular mechanisms and personalized medicine meeting report. Int J Gynecol Cancer 2012; 22: S45-57.

3. Suh DH, Kim HS, Chang SJ, Bristow RE. Surgical management of recurrent ovarian cancer. Gynecol Oncol 2016; 142: 357-367.

4. Falcone F, Scambia G, Benedetti Panici P, et al. Tertiary cytoreductive surgery in recurrent epithelial ovarian cancer: A multicentre MITO retrospective study. Gynecol Oncol 2017; 147: 66-72.

5. Wyler S, Huang DJ, Singer G, et al. Metastatic involvement of the urinary tract in patients with advanced ovarian carcinoma: lessons from the autopsy for an interdisciplinary treatment approach. Eur J Gynaecol Oncol 2009; 30: 174-177.

6. Nishikimi K, Tate S, Matsuoka A, Shozu M. Complete resection of locally advanced ovarian carcinoma fixed to the pelvic sidewall and involving external and internal iliac vessels. Gynecol Oncol 2017; 146: 436-437.

7. Cascales PA, Gil J, Alarcón CM, et al. Urinary tract surgery in patients with ovarian peritoneal carcinomatosis treated with cytoreduction and hyperthermic intraoperative intraperitoneal chemotherapy. Cir Esp 2012; 90: 162-168.
8. Pujade-Lauraine E, Ledermann JA, Selle F, et al. Olaparib tablets as maintenance therapy in patients with platinum-sensitive, relapsed ovarian cancer and a BRCA1/2 mutation (SOLO2/ENGOT-Ov21): a double-blind, randomised, placebo-controlled, phase 3 trial. Lancet Oncol 2017; 18 : 1274-1284.

9. Lee JM, Nair J, Zimmer A, et al. Prexasertib, a cell cycle checkpoint kinase 1 and 2 inhibitor, in BRCA wild-type recurrent high-grade serous ovarian cancer: a first-in-class proof-of-concept phase 2 study. Lancet Oncol 2018; 19: 207-215.

10. Rekhi B, Deodhar KK, Menon S, et al. Napsin A and WT 1 are useful immunohistochemical markers for differentiating clear cell carcinoma ovary from high-grade serous carcinoma. APMIS 2018; 126: 45-55.

11. Sehouli J, Grabowski JP. Surgery for recurrent ovarian cancer: Options and limits. Best Pract Res Clin Obstet Gynaecol 2017; 41: 88-95.

12. Fanfani F, Fagotti $A$, Ercoli $A$, et al. Is there a role for tertiary (TCR) and quaternary (QCR) cytoreduction in recurrent ovarian cancer? Anticancer Res 2015; 35: 6951-6955.

13. Hızlı D, Boran N, Yılmaz S, et al. Best predictors of survival outcome after tertiary cytoreduction in patients with recurrent platinum-sensitive epithelial ovarian cancer. Eur J Obstet Gynecol Reprod Biol 2012; 163: 71-75.

14. Fotopoulou C, Richter R, Braicu IE, et al. Clinical outcome of tertiary surgical cytoreduction in patients with recurrent epithelial ovarian cancer. Ann Surg Oncol 2011; 18: 49-57. 